\title{
Computer simulation of photoinduced molecular motion and reactivity
}

\author{
Marco Garavelli, ${ }^{1}$ Fernando Bernardi, ${ }^{1}$ Michael A. Roob, ${ }^{2}$ and Massimo Olivucci ${ }^{3}$ \\ ${ }^{1}$ Dipartimento di Chimica “G. Ciamician”, Università di Bologna, Via Selmi 2, 40126 Bologna, Italy \\ ${ }^{2}$ Department of Chemistry, King's College London, Strand, London WC2R 2LS, United Kingdom \\ ${ }^{3}$ Istituto di Chimica Organica, Università di Siena, Via Aldo Moro, I-53100 Siena, Italy
}

\begin{abstract}
The use of the computer to simulate light induced chemical processes has given access to a detailed description of the molecular motion and mechanism underlying the reactivity of organic and bio-organic chromophores. Thus, different computational strategies and tools can now be operated like "virtual spectrometers" to characterize the photoinduced molecular deformation and reactivity of a given chromophore. Furthermore, a systematic computer investigation of a wide range of photochemical organic reactions during the last decade, has lead to novel concepts that allow the chemist to formulate the mechanism of a photochemical reaction in a rigorous way and with a language which is familiar to chemists.

In this review we will revise some basic mechanistic concepts which have emerged in the novel field of computational photochemistry. These comprise the concept of conical intersection funnels, photochemical paths, path branching and selectivity. Recent work in the field of biological photoreceptors has demonstrated that computational chemistry can be successfully applied to photobiological problems. Here we will revise the results of the photoisomerization path mapping of the protonated Schiff base of retinal, the chromophore of rhodopsin proteins. These studies have produced the "two-state two-mode model" which provides a description of the photon-induced molecular motion in the isolated retinal chromophore. Such model represents a substantial revision of the previous models for the primary event in vision in animals and light driven proton-pumping in halobacteriae.
\end{abstract}

\section{INTRODUCTION}

A detailed understanding of the reaction pathways evolving in the excited state manifold may increase our ability to design new and to control known photochemical reactions. Among these, many intriguing photobiochemical processes involve conjugated $\pi$-systems. Examples are the set of photochemical reactions characterizing the bio-genesis of vitamin $\mathrm{D}_{2}$ [1] (as well as the properties of photochromic materials [2]) and the conversion of light into chemical energy in plants and animals, which involves extended conjugated molecules, such as carotenoids and retinals, bound in protein complexes [3]. The use of such extended systems in optical data storage and processing technology is now being investigated.

Photobiological systems such as rhodopsin proteins (a family of photoreceptors) exploit the ability of retinal chromophores to undergo cis/trans photoisomerization and to transduce radiative energy into thermal (i.e. kinetic) energy on picosecond or shorter timescales [4]. Recent advances in time-resolved spectroscopy (e.g. the use of ultrafast laser pulses) has provided a powerful tool to monitor reaction dynamics on the femtosecond timescale [5] and made direct observation of these processes possible, increasing our understanding of the excited state structures and dynamics for model systems. Ultrafast (femtosecond) radiationless decay has been observed, for example, for simple dienes [6], cyclohexadienes [7, 8], hexatrienes [9], and in both free [4](a) and opsin-bound [4](b) retinal protonated Schiff bases (RPSBs). However, a complete understanding of molecular dynamics on the excited state manifold is required to interpret these laser experiments with confidence and to understand the principles involved in the design of optical devices. Computational chemistry may now represent a tool for such a detailed investigation, and in this review we will outline the strategy and operational approach to the practical computational investigation of photochemical reaction mechanisms. As an example, application to the intriguing problem of photoinduced cis/trans isomerization in RPSBs will be shown, and the recent advances in this area will be revised.

\section{MODELING PHOTOCHEMICAL REACTIONS: GENERAL ASPECTS}

Until recently, reaction path computations were limited to the investigation of thermal reactions or, in general, to reactions occurring on a single potential energy surface (PES). For such a process, state-of-the-art $a b$ initio quantum chemical methods provide a complete description of what happens at the molecular level during the bond-breaking bond-forming processes. In particular, it is possible to compute the transition structure which connects a reactant to a product and the associated energy barrier with nearly chemical accuracy (ca. $1 \mathrm{kcal} \mathrm{mol}^{-1}$ error). Furthermore, the reaction path, i.e. the progression of the molecular structure towards the 
transition state and the product, can be determined, in a fully unbiased way, by computing the minimum energy path (MEP) [10] connecting the reactant to the product along the $3 \mathrm{~N}-6$ dimensional potential energy surface of the system.

Photochemical processes, where, typically, the reactant resides on an excited state potential energy surface and the products accumulate on the ground state could not be easily investigated. In fact, the reaction path is expected to have two branches; one located on the excited state and the other located on the ground state energy surface. The main difficulty associated with such a computation lies in the correct definition and practical computation of the "funnel" where the excited state reactant or intermediate is delivered to the ground state. While the progression on the excited state energy surface (i.e. the excited state "branch" of the reaction path) may be investigated with the same type of methods used for thermal reactions, a general way of defining the "locus" where this reaction path branch would be connected to the ground state branch was lacking. During the last decade, computational chemists have been able to develop tools and learn strategies to explore electronically excited state species [11]. The goal of such computational approaches, in the study of photochemical mechanisms, is the complete description of what happens at the molecular level from energy absorption to product formation (i.e. the full description of the reaction path). Here, we will focus on the new theoretical tools (which have been partly developed by our group), with the case study of retinal chromophores, to show how they can be applied.

\subsection{Conical intersections in photoinduced pro-} cesses. As a result of an intensive computational and experimental work, new aspects of the behavior of photoexcited organic molecules have emerged which have allowed for a more systematic investigation of photochemical reaction pathways. The most general of these is that the nature of the funnel, i.e. the joint point between the two branches of the photochemical path and the locus where decay from the excited to the ground state occurs, has been clarified. In general, this point takes the form of a low-lying intersection. In fact, it has been shown that low-lying intersections (crossings) between the photochemically relevant excited state and the ground state occur with a previously unsuspected frequency $[11](f),(g)$. Thus, an excited state intermediate during its motion has a high probability of entering a region where the excited state crosses the ground state. Therefore, such crossings, i.e. conical intersections in the case of two singlet (or two triplet) states, or singlettriplet intersections, provide a very efficient "funnel" for radiationless deactivation (internal conversion or intersystem crossing) which may occur in a single molecular vibration (picosecond or subpicosecond timescale) and, in turn, prompt photoproduct formation.
The rate and the energy thresholds controlling internal conversion (IC) can now be experimentally measured by exploiting the advances in laser spectroscopy, which have pushed the time resolution of various experimental techniques below the picosecond timescale. Several results indicate that the Van der Lugt and Oosteroff model [12] for IC has to be refined by replacing the classic picture of an "avoided crossing" with that of an "unavoided crossing", (e.g. a conical intersection, see Scheme 1), thus confirming computational predictions. Indeed, femtosecond excited state lifetimes observed in many conjugated systems [4, 6-9] are in agreement with a decay via a real crossing, while IC via "avoided crossing" would occur on a timescale of several molecular vibrations ( $\mu$ s to ns), i.e. much slower and close to that of fluorescence.
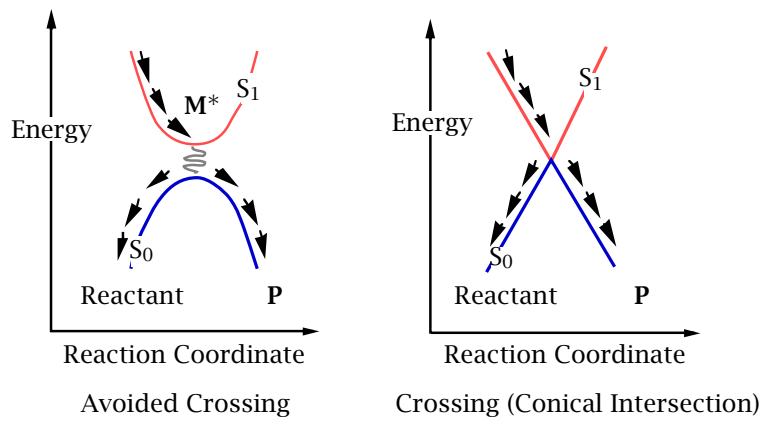

Scheme 1.

A view of photochemical reactions, that is consistent with recent experiments, was suggested more than 30 years ago by the physicist Edward Teller [13] at the $20^{\text {th }}$ Farkas Memorial Symposium. He suggested that it was the electronic factors that may play the dominant role in the efficiency of radiationless decay. Teller made two general observations: (i) in a polyatomic molecule the non-crossing rule, which is rigorously valid for diatomics, fails and two electronic states, even if they have the same symmetry, are allowed to cross at a conical intersection; (ii) radiationless decay from the upper to the lower intersecting state occurs within a single vibrational period when the system "travels" in the vicinity of such intersection points. On the basis of these observations, Teller proposed that conical intersections may provide a common and very fast decay channel from the lowest excited states of polyatomics. In the field of photochemistry, Zimmerman [14] and Michl [15] were the first to suggest, independently, that certain photoproducts originate from IC at a conical intersection. Zimmerman and Michl use the term "funnel" for this feature.

The suggestions of Teller, Zimmerman and Michl have been fully supported by recent computational work [11](f)-(h). When taken in conjunction with modern experimental results, this indicates that the 
radiationless decay process may very often occur via a conical intersection between the excited and ground states. Radiationless decay at a conical intersection implies that: (a) the IC process will be $100 \%$ efficient (i.e. the Landau-Zener [11](c),(e) decay probability will be unity), (b) any observed retardation in the IC or reaction rate (i.e. the competition with fluorescence) must reflect the presence of some excited state energy barrier which separates the excited state intermediate $\mathbf{M}^{*}$ from the intersection structure (Figure 1) and (c) in the case where the decay leads to a chemical reaction, the molecular structure at the intersection must be related to the structure of the photoproducts $(\mathbf{P})$, as in thermal reactions the structure of the transition state is related to that of the products.

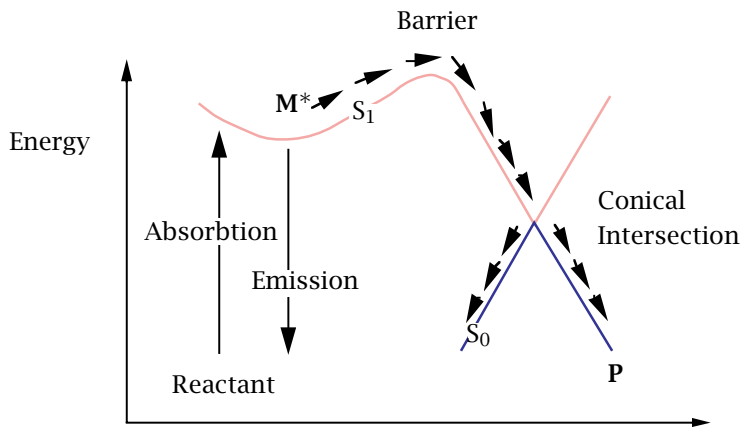

Reaction Coordinate

Figure 1. Schematic reaction path for a barrier controlled "opening" of a fast radiationless decay channel.

2.2. Computational modeling. Points a-c presented above provide the theoretical basis for the computational modeling of photochemical reactions. According to this approach, the excited state motion is determined by the structure of the relevant excited and ground state potential energy surfaces. In simple terms, information on the excited state lifetime and on the type of photoproducts generated is obtained by following the detailed relaxation and reaction paths of the molecule along the reaction coordinate onto the involved potential energy surfaces, from the FranckCondon point, or excited state intermediate $\left(\mathbf{M}^{*}\right)$, to the ground state. Accordingly, the strategy used in computational photochemistry is based on the mapping of the photochemical reaction path computed by following the MEP from the starting (e.g. Franck-Condon structure FC) to the final (e.g. ground state photoproducts) points through, for example, a conical intersection CI (see Scheme 2). This method (recently named pathway approach by Fuss et al. [16]) is part of a more general way of considering photochemistry which is already employed in textbooks [11](c): it follows the pathway on the potential energy surfaces paying attention to local details and properties like slopes, saddle points (TS), barriers and funnels (such as conical intersections). This approach is now receiving new impact by recent advances in femtosecond spectroscopy and ultrafast techniques and has an intimate connection to the method using the motion of wavepackets or semiclassical trajectories on potential surfaces to describe ultrafast photochemical processes (Scheme 2).

\subsection{MEP mapping versus standard geometry op-}

timization. The strategy outlined above provides information on the structure and accessibility of the photochemical reaction paths (i.e. MEPs in computational terms) from a chosen starting point (e.g. the FC point). This technique has the advantage of "self-limiting" the investigation only to the portion of the PES which is relevant for the description of the photo-process. In other words, by following the MEP, we immediately focus onto the driving forces responsible for the photoinduced motion. Therefore, only those intermediates, transition states, and funnel points which are directly accessible by the system, will be located as "travelingpoints" along the MEP. Many other stationary points and crossing regions (which may be located, in principle, via simple geometry optimizations) may be very far from the followed reaction channels (MEPs), both in energies and geometries, and are not important for the description of the process. Thus, for example, the radiationless decay (funnel) point intercepted by the MEP may be very different from the optimized lowerenergy one. This explains why the information given by the MEP may be quite different (and complementary) from the one provided by locating stationary points and lower-energy funnel regions (which may account for a more general and extended description of the shape of the PES). We will give examples of that in Section 4.

2.4. Topology versus dynamics. The pathway approach provides a structural (i.e. non-dynamical) information. Since molecules have more than infinitesimal kinetic energy, a classical trajectory will not follow the MEP and may in fact deviate quite widely from it such as in the case of a "hot" system (i.e. with a high excess vibrational energy). In this case, also other portions of the PES (i.e. far and higher energy stationary points) may become important and a dynamics treatment of the motion on the PES would be required. However, the MEP may be a convenient measure of the progress of a molecule in a reaction since, in general, a molecule will move (on the average) along the MEP in a well defined valley. Moreover, it is a good approximation of the motion of vibrationally cold systems (e.g. for photochemical reactions where the excited state reactant has a small/controlled amount of vibrational excess energy), providing insight into the mechanism of photoproduct formation from vibrationally "cold" excited state reactants, such as those encountered in many experiments where slow excited state motion or/and thermal 


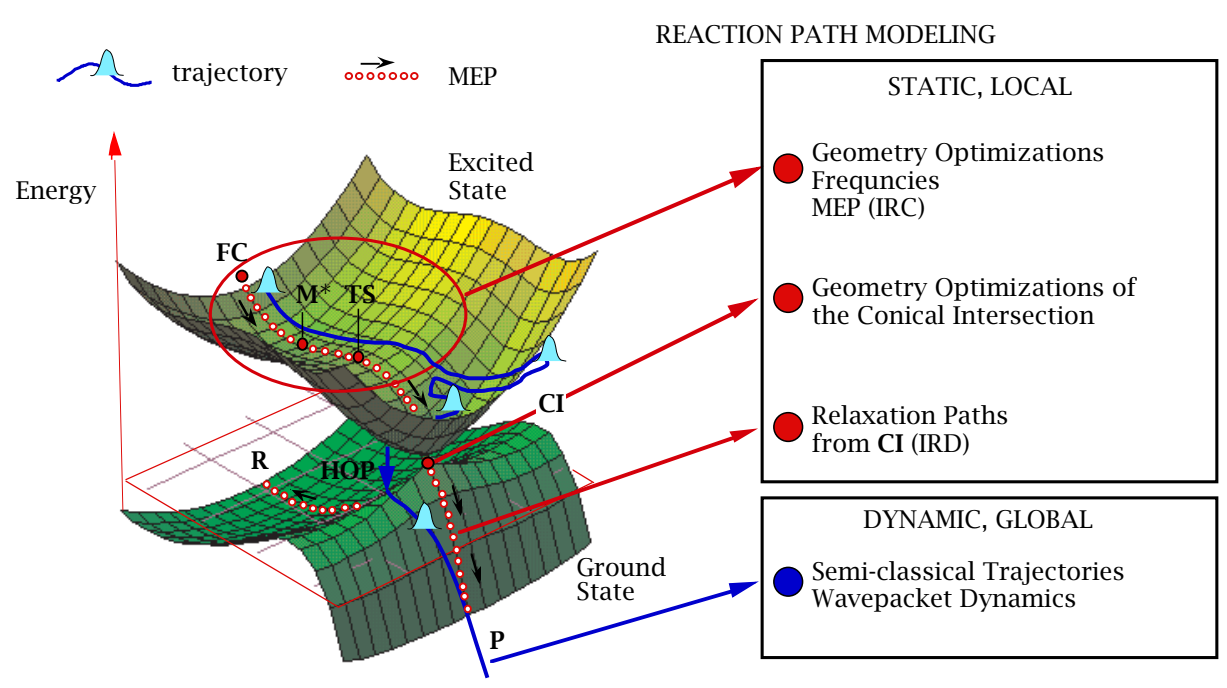

Scheme 2.

equilibration is possible (e.g. in cool jets, in cold matrices and in solution). Under these conditions of low vibrational excess energy, semi-classical dynamics yields the same mechanistic information as from topological investigation of the PES, because its structure is expected to play the dominant role in determining molecular motion (see the MEP line versus the real trajectory in Scheme 2).

2.5. Models selection. During the last decade or so, the speed of the computers has grown considerably and the computational investigation of realistic models of organic compounds is becoming a standard practice. Nevertheless, the theoretical chemist who wants to perform accurate computational investigations of photobiochemical processes (such as the ones involving RPSBs), faces a target-size related problem. To apply accurate computational methods, it is necessary to reduce the dimension of the molecule, by selecting a model-system with the same chemical reactivity but a smaller size. It is usually a good approximation to limit our study only on the polyenic moiety of the system: this is the chromophore responsible of light absorption and, in general, the overall molecule reactivity is driven by the reactivity of this sub-unit. Moreover, the reduced size of the system (provided it is small enough) makes it possible to perform very accurate computations. In Scheme 3 we show how retinal models $(1,2$, and 3 ) have been selected according to the nature and accuracy of the computational results we want to obtain.

\section{COMPUTATIONAL TOOLS}

Modeling techniques that are used in studying photochemical mechanisms are limited to those that can describe excited states correctly. In principle, every method which allows the correct computation of excited states energy and its first (i.e. the gradient) and second (i.e. the Hessian) derivatives may be used for this purpose. Here, we just say that all the geometry optimizations used to get the results presented in this review (i.e. for locating minima, transition states, conical intersection points and for computing MEPs) have been obtained inside the framework of the ab initio CAS-SCF method [11](d). This is the main multi-reference wavefunction method used for geometry optimization of excited states since it permits the gradient and second derivatives to be computed analytically and has been implemented in widely distributed programs such as Gaussian 98 [17] and MOLCAS 4 [18]. For the studied models $(1,2$, and 3$)$, the active space used comprises the whole space of $\pi$ electrons and $\pi$ orbitals, and a $6-31 \mathrm{G}^{*}$ basis set.

When accurate energetics are required, a treatment of dynamic correlation and the MP2 method (i.e. the multireference-MP2 method) has been implemented [19] within the context of CAS-SCF theory, and this gives reliable results (i.e. errors within $3 \mathrm{kcal} / \mathrm{mol}$ ) at modest cost. All the energies presented in this review have been refined at the multireference-MP2 level using the CAS-PT2 method included in MOLCAS 4 [18].

Standard methods for molecular structure optimization of stationary points (i.e. reactants, products, intermediates, and transition states) as well as for MEP computations (which can be done by using the intrinsic reaction co-ordinate (IRC) [20] method) are widely available both for ground or excited states. However, the description of the crossing region requires special methods because the Born-Oppenheimer approximation breaks down. Nowadays, methodologies for locating and optimizing crossings between two potential energy surfaces are also public; for example, these have been implemented in the Gaussian 98 [17] suite of programs. All these methods have already been widely 
11-cis RETINAL CHROMOPHORE (PSB11)

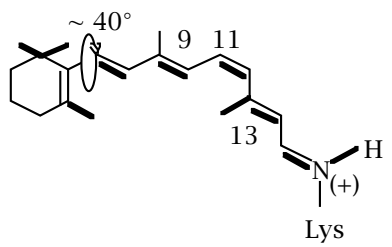

MODELS
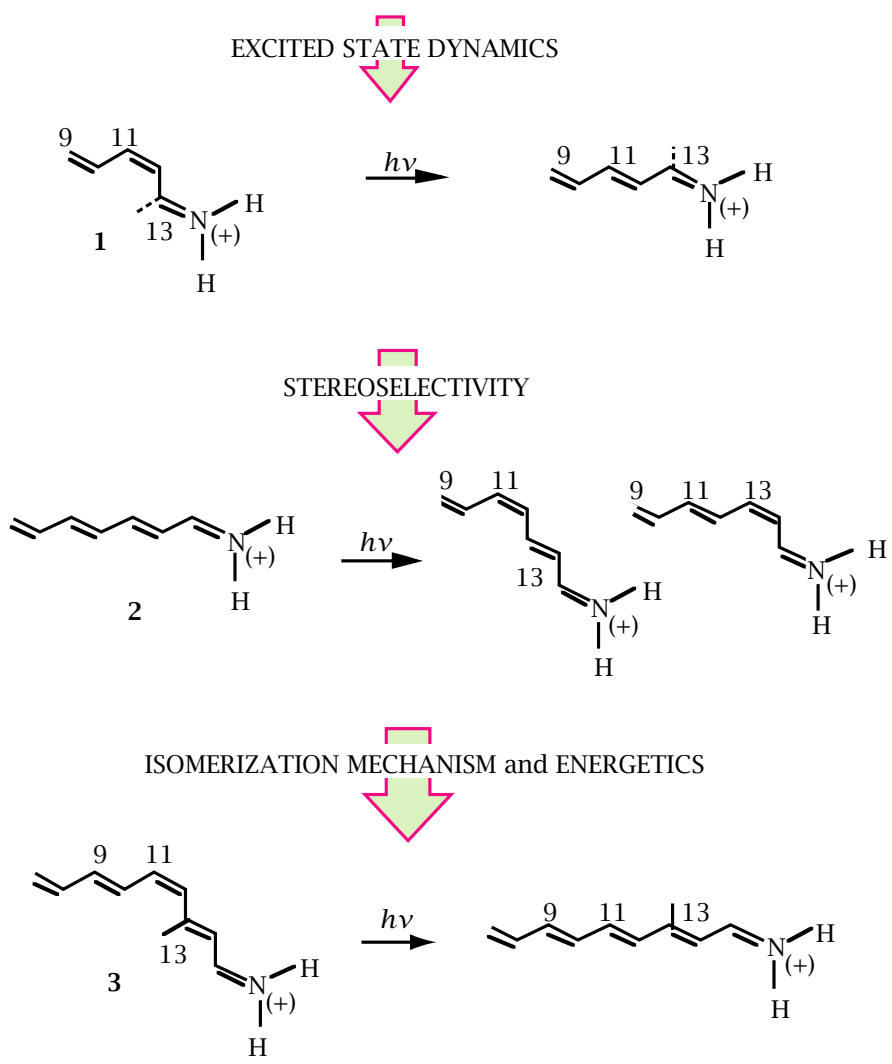

Scheme 3.

discussed in previous reviews [21] and won't be presented here.

Here, we will focus on the branching process involving the photochemical path upon decay from a higher to a lower laying state. The inter-state nature of photochemical reaction paths requires special methodologies to locate the energy valleys describing the branching process itself (e.g. ground state relaxations occurring after the decay at the crossing). Methods for computing relaxation paths starting from a crossing point (or, more generally, from non-stationary points) are still matter of research and to our knowledge are not publicly available.

\subsection{Locating decay paths from a conical intersec-}

tion. We have shown that an accessible conical inter- section forms a bottleneck that separates the excited state branch of a non-adiabatic photochemical process from the ground state branch, thus connecting the excited state reactant to two or more products on the ground state via a branching of the excited reaction path into several ground state relaxation channels. The nature of the products generated following decay at a surface crossing will depend on the ground state valleys (reaction paths) that can be accessed from that particular structure. We have recently implemented a gradient-driven algorithm [22] to locate and characterize all the relaxation directions departing from a single conical intersection point. The MEP starting along these relaxation directions define the ground state valleys, which determine the possible relaxation processes and the photoproducts. Thus, a connection between excited 
state and ground state reaction paths and a full description of the photochemical process from energy absorption to photoproduct formation may be achieved.

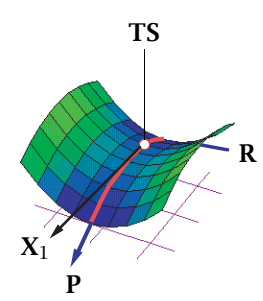

(a)

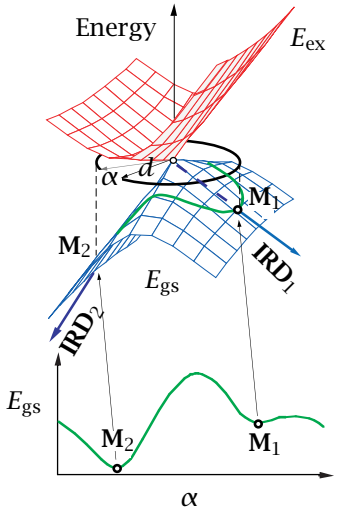

(b)
Figure 2. (a) Model PES showing a transition state (TS) connecting a reactant $(\boldsymbol{R})$ to a product $(\boldsymbol{P})$ in standard reactivity. (b) PES for a "model" elliptic conical intersection, and the corresponding energy profile (as a function of the angle $\alpha$ ) along a circular cross section centered on the conical intersection point and with radius $d$.

The MEP connecting the reactant (R) to the product $(\mathbf{P})$ of a thermal reaction is uniquely defined by the associated transition structure (TS). The direction of the transition vector (i.e. the normal co-ordinate corresponding to the imaginary frequency of the TS) is used to start a MEP computation. One takes a small step along this vector $\mathbf{x}_{1}$ (shown in Figure 2(a)) to points $\mathbf{R}$ or $\mathbf{P}$ and then follows the steepest descent paths connecting this point to the product or reactant well. The small step vector defines the initial relaxation direction (IRD) towards the product or reactant. This procedure cannot be used to find the IRD for a photochemical reaction since, as discussed above, a conical intersection is a "singularity" and there is no such unique direction for this first step (i.e. a frequency computation cannot be performed at a conical intersection point). The general situation is illustrated in Figure 2(b) for an elliptic cone (i.e. linear approximation). In this case, two steep sides exist in the immediate vicinity of the apex of the cone (see Figure 2(b)). It is thus obvious that there are two preferred directions for downhill motion located on these steep sides of the ground state cone surface. As one moves away from the apex along these steep directions, real reaction valleys eventually develop (leading to the final photoproduct minima). A simple procedure for defining these directions involves the computation of the energy profile along a circular cross-section centered on the vertex of the cone. This energy profile is given in Figure 2(b) as a function of the angle $\alpha$ and for a suitable choice of the radius $d$. It can be seen that the profile contains two different energy minima. These minima ( $\mathbf{M}_{1}$ and $\mathbf{M}_{2}$ in Figure 2(b)) uniquely define the two IRDs $\left(\mathbf{I R D}_{1}\right.$ and $\left.\mathbf{I R D}_{2}\right)$ from the vertex of the cone. The two steepest descent lines starting at $\mathbf{M}_{1}$ and $\mathbf{M}_{2}$ define two MEPs describing the relaxation processes in the same way in which the transition vector $\mathbf{x}_{1}$ (see Figure 2(a)) defines the MEP connecting reactants to products. Thus, while there is no analogue of the transition vector for a conical intersection, the simple case of an elliptic cone shows that the IRD are still uniquely defined in terms of $\mathbf{M}_{1}$ and $\mathbf{M}_{2}$. Notice that while the IRD from a TS connects the reactant to the product, there are two IRD from an elliptic conical intersection leading to two different photoproduct valleys (and one of these photoproducts may correspond to the original reactant).

Although this model of the potential energy sheets at a conical intersection point is not general enough to give a correct description of all the relaxation paths encountered in a real system, however the ideas introduced above can be easily extended to search for IRD in the full $n$-dimensional space surrounding a conical intersection point by replacing the circular cross-section with a (hyper)spherical cross-section centered at the vertex of the cone. Thus, locating stationary points on the $n-1$ dimensional hypersphere involves constrained geometry optimization, in mass-weighted co-ordinates, with a "frozen" variable $d$ (i.e. the radius). The full mathematical details have been presented elsewhere [22].

We must emphasize that the procedure outlined above is designed to locate the points where the relaxation paths begin (i.e., they define the IRD). Once these points have been found for some small value of $d$, then one must compute the associated MEP (via using the standard intrinsic reaction co-ordinate (IRC) [20] method) which defines the relaxation paths leading to ground state energy minima (as stated before). Thus the approach outlined above provides a systematic way to find the MEP connecting the vertex of the cone to the various ground state photoproduct wells. Since more than one MEP originates from the same conical intersection point, this procedure also describes the branching of the excited state reaction path occurring at the intersection point.

Although this methodology has been presented for the case of the ground state reaction paths departing from a conical intersection, it may be used as well for locating relaxation channels from a different nonstationary point (e.g. the FC point).

\section{A CASE STUDY: THE PROBLEM OF RETINAL CHROMOPHORE PHOTOISOMERIZATION}

An application of the computational strategy outlined above is shown in Figure 3, which illustrates the evolution of the "minimal" retinal chromophore model 1 (the cis- $\mathrm{C}_{5} \mathrm{H}_{6} \mathrm{NH}_{2}{ }^{+}$isomer) along the interstate MEP 


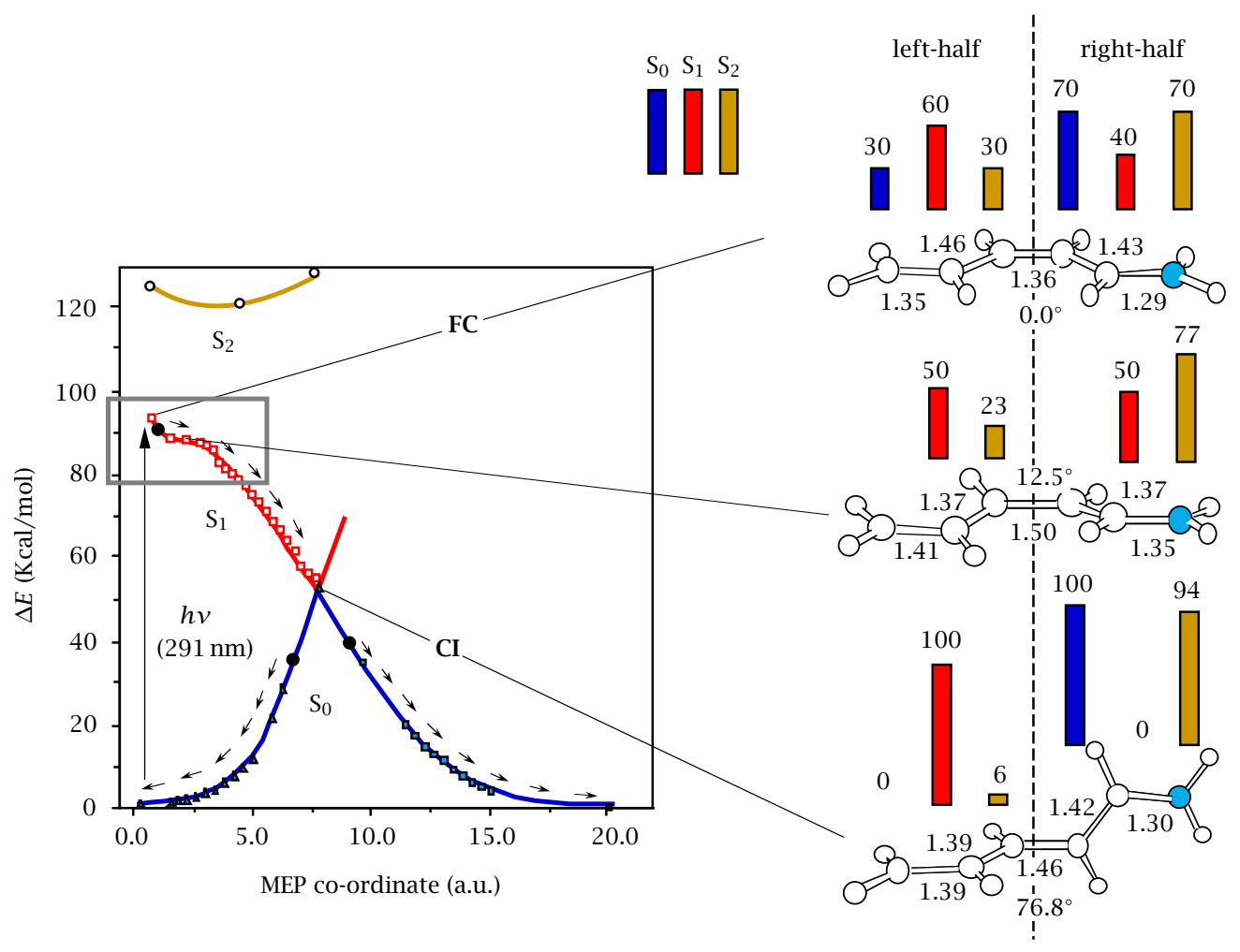

Figure 3. Energy profiles along the three MEPs describing the relaxation from the Franck-Condon (FC) and conical intersection (CI) points. Open and full squares define the excited (1Bu-like) and ground state branches of the cis $\rightarrow$ trans photoisomerization path, respectively. Full triangles define the ground state cis back-formation path. Open circles show the dark (2Ag-like) state energy along the excited state branch of the photoisomerization path. The structures (geometrical parameters in $A$ and degrees) document the geometrical progression along the photoisomerization path, while the bar diagrams describe the change in the $\mathrm{S}_{0}, \mathrm{~S}_{1}$ and $\mathrm{S}_{2}$ Mulliken charges (as \% unit of the net positive charge) of the $\mathrm{C}=\mathrm{C}-\mathrm{C}-($ left) and $-\mathrm{C}-\mathrm{C}=\mathrm{N}$ (right) fragments.

connecting the FC structure of the cis isomer to the $\mathrm{S}_{0}$ trans and cis product wells [23]. The solid circle on $\mathrm{S}_{1}$ locates the first computed IRD, which defines (as illustrated above) the starting point of the excited state MEP from the $\mathbf{F C}$ region.

Following photoexcitation to the spectroscopic (i.e. ionic 1Bu-like) $S_{1}$ state, the computed MEP defines the photoisomerization of the central double bond, which occurs along a fully barrierless reaction path. Along this path, the energy difference between the $S_{1}$ and $S_{2}$ (covalent $2 \mathrm{Ag}$-like) states is large $\left(>25 \mathrm{kcal} \mathrm{mol}^{-1}\right)$ and thus it appears that the covalent $S_{2}$ is never involved during the reaction. Moreover, evolution of the charges along the reaction coordinate (see Figure 3 ) shows an increasing ionic character for the $S_{1}$ state which is consistent with the increasing $S_{1} / S_{2}$ energy separation along the MEP and the consequent smaller mixing between these two states. The $S_{1}$ MEP ends at a point where $S_{1}$ and $S_{0}$ cross at a conical intersection (CI). This intersection point $(\mathbf{C I})$ has $\mathrm{a} \sim 80^{\circ}$ twisted central double bond which provides a route for a fully efficient radiationless decay and non-adiabatic cis $\rightarrow$ trans isomer- ization. Interestingly, the CI structure corresponds to a twisted intramolecular charge transfer (TICT) state: a single $\pi$-electron has smoothly migrated, along the $\mathrm{S}_{1}$ MEP, from the left $\mathrm{CH}_{2}-\mathrm{CH}-\mathrm{CH}-$ allyl fragment to the right $-\mathrm{CH}-\mathrm{CH}-\mathrm{NH}_{2}$ fragment and, consequently, the positive charge is almost completely delocalized on the ally fragment. At the degenerate $S_{0}$ state, the electron shifts back to the left side and the original $S_{0}$ charge distribution is restored. Starting from the crossing point we have located two IRD on $\mathrm{S}_{0}$ (see the two solid circles close to the $\mathbf{C I}$ ), where two corresponding relaxation paths begins (MEPs). The first path is a continuation of the excited state path and ends at the alltrans $\mathrm{C}_{5} \mathrm{H}_{6} \mathrm{NH}_{2}{ }^{+}$photoproduct well. The second path describes the back-formation of the reactant. In summary, such a computational approach has lead to a detailed description of what happens in model $\mathbf{1}$ along the reaction coordinate from absorption to photoproduct formation. The photoisomerization process is thus fully characterized.

The same computational strategy has been extended to the study of the photoisomerization of longer PSBs 


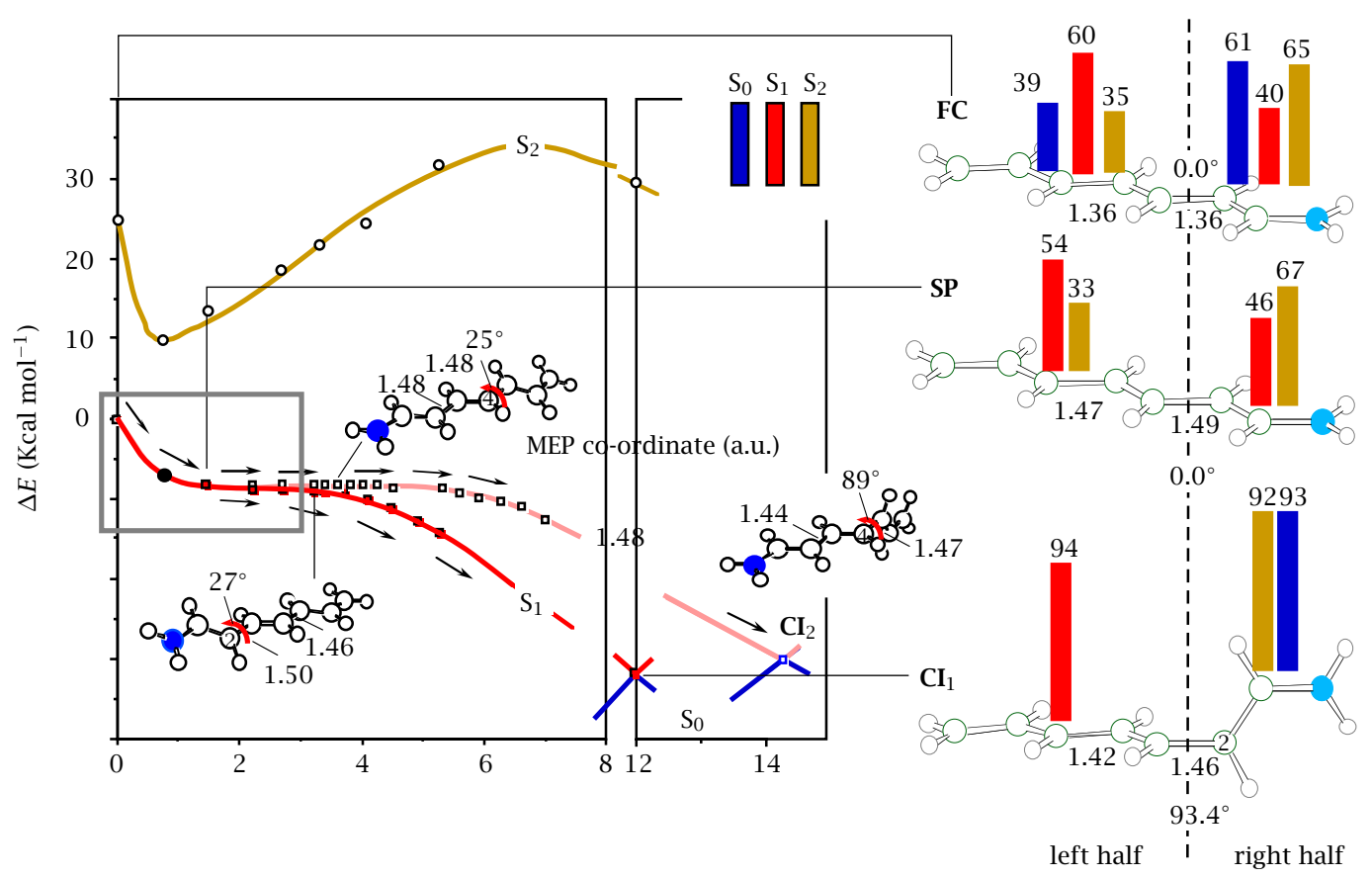

Figure 4. Energy profiles along the MEPs describing the two competing (open and full squares) excited state (1Bu-like) isomerization paths from the Franck-Condon point (FC) to the decay points (conical intersections) $\boldsymbol{C I}_{1}$ and $\mathbf{C I}_{2}$ of all-trans $\mathrm{C}_{7} \mathrm{H}_{8} \mathrm{NH}_{2}{ }^{+}$. The relaxed planar stationary point is labeled $\boldsymbol{S P}$. Open circles show the dark (2Ag-like) state energy along the $\mathrm{S}_{1}$ branch of the favored photoisomerization path. The structures (geometrical parameters in $\AA$ and degrees) document the geometrical progression along the two paths, while the bar diagrams describe the change in the $\mathrm{S}_{0}, \mathrm{~S}_{1}$ and $\mathrm{S}_{2}$ Mulliken charges (as \% unit of the net positive charge) of the $\mathrm{C}=\mathrm{C}-\mathrm{C}=\mathrm{C}-\mathrm{C}-$ (left) and $-\mathrm{C}-\mathrm{C}=\mathrm{N}$ (right) fragments.

such as 2 and 3, leading to nearly the same qualitative results [23](b),(c), as illustrated in Figures 4 and 5. While model 2 allows us to investigate competitive photoisomerization channels, model 3 provides a more realistic description of what happens for the real retinal chromophore (RPSB) in isolated conditions. Thus, model 3 is the best candidate for accurate energetics and for a comparison with experimental data and observations (see Scheme 1).

Again, a solid circle close to the FC point (see Figures 4 and 5) locates the IRD where a barrierless or nearly barrierless MEP starts. Again, the $\mathrm{S}_{1}$ state preserves its ionic (charge-transfer) nature all along the excited state MEP, which eventually ends at a CI point (between $\mathrm{S}_{1}$ and $\mathrm{S}_{0}$ ) corresponding again to a TICT state with an almost $90^{\circ}$ twisted central double-bond (see charge evolution along the MEP as illustrated in Figures 4 and 5). However, in contrast to 1 , longer PSB undergo competitive trans/cis photoisomerizations involving internal double bonds (e.g. the two double bonds in position 2 and 4 for model 2). The presence of competing channels that are barrierless or nearly barrierless is an interesting feature of longer PSBs. Here, we have characterized the $S_{1}$ MEPs of both these competing isomerization processes for model 2 , as illustrated in Figure 4 where we have reported the two corresponding unconstrained MEPs. The two paths are nearly barrierless with only a $<1 \mathrm{kcal} \mathrm{mol}^{-1}$ energy difference along the flat energy region (see framed region in Figure 4) in favor of the isomerization at position $\mathrm{C}_{2}-\mathrm{C}_{3}$.

From inspection of the reaction coordinate, another interesting feature emerges, which is in common among all the studied PSB models (1, 2 and 3): the initial part of the MEP is dominated by an in-plane mode corresponding to double-bonds expansion/single-bonds contraction. Thus, the FC structure initially evolves along a totally symmetric stretching mode, and the results of analytical frequency computations confirm that the structure of the potential energy surface around the FC region is that of a steep valley with a slope (i.e. a gradient) driving the system along a planar backbone rearrangement. Only after this initial symmetric motion, when the system approaches the relaxed planar stationary point (SP) where bond order inversion has occurred, the reaction coordinate curves and an asymmetric twisting mode (i.e. the isomerization mode about the twisting double bond) starts to dominate the process. In fact, the reaction channel (coming from the FC point) changes its shape and the valley evolves (via an inflection point) into a ridge at SP (as analytical frequency computations show [23](b)). Figure 6(a)-(c) illustrates a schematic view for the structure of the $S_{1}$ 


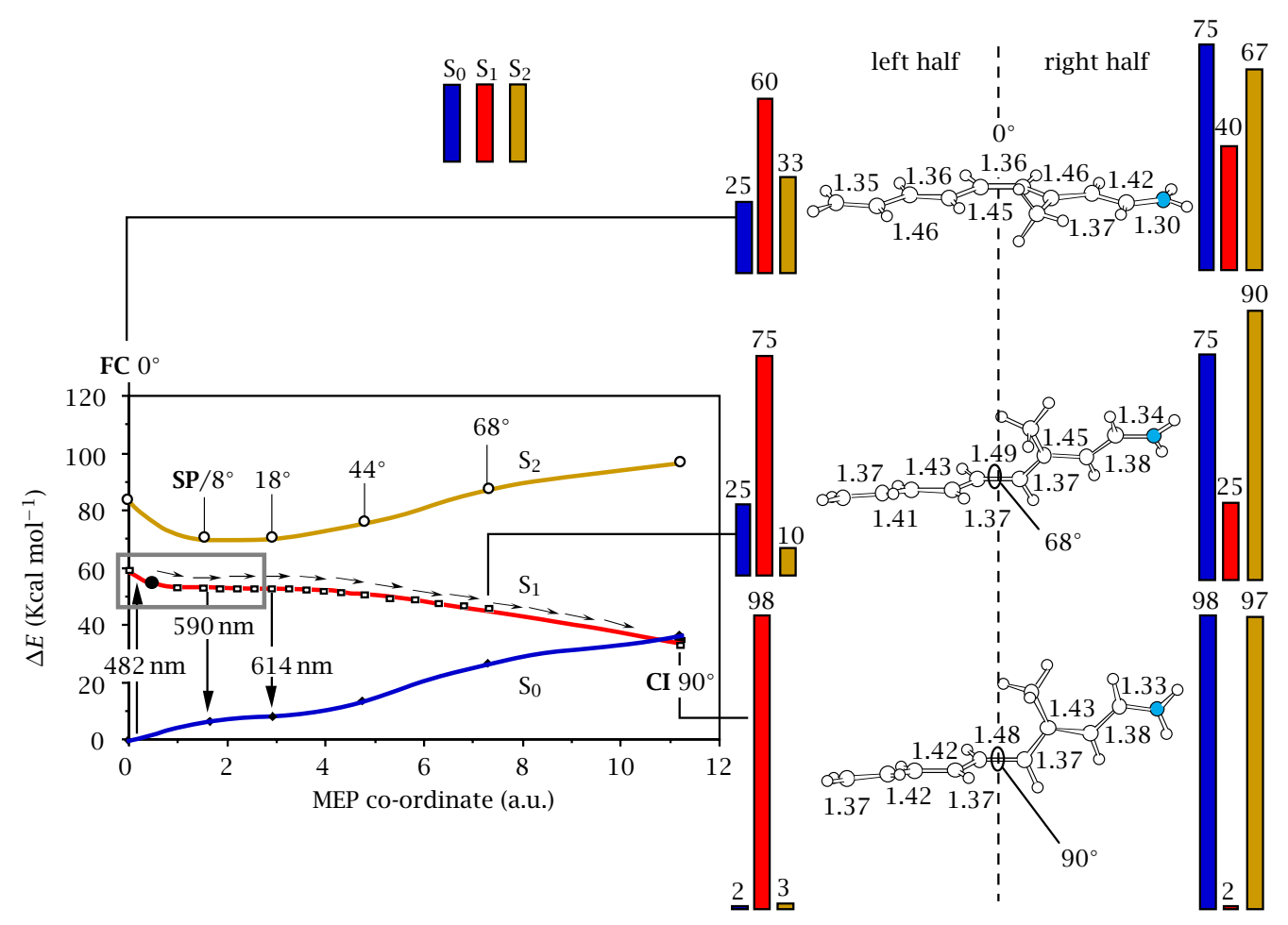

Figure 5. Energy profiles along the $\mathrm{S}_{1}$ reaction coordinate of the PSB11 model 1 (open squares) from the Franck-Condon point (FC) to the decay point $\mathbf{C I}$ (conical intersection). Open circles and diamonds show the $\mathrm{S}_{2}$ and $\mathrm{S}_{0}$ energy cross-sections along the same coordinate. The structures (geometrical parameters in A and degrees) document the progression of the molecular structure along the coordinate, while the bar diagrams describe the change in the $\mathrm{S}_{0}, \mathrm{~S}_{1}$ and $\mathrm{S}_{2}$ Mulliken charges (as \% unit of the net positive charge) of the $\mathrm{C}=\mathrm{C}-\mathrm{C}=\mathrm{C}-\mathrm{C}-$ (left) and $-\mathrm{C}-\mathrm{C}=\mathrm{C}-\mathrm{C}=\mathrm{N}$ (right) fragments.

PES for 1, 2 and 3 respectively. Thus, for example, the two competing isomerization paths found in model 2 (see Figure 4) have a common initial part, which is dominated by double-bond expansion. Evolution along the torsional co-ordinate (and therefore either of the two competing paths to $\mathbf{C I}_{1}$ and $\mathbf{C I}_{2}$ ) begins only after relaxation in the vicinity of the planar stationary point SP, where the MEP bifurcates (see Figure 6(b)). Initial relaxation along a totally symmetric mode followed by motion along non totally symmetric (twisting) modes seems to be a general feature of the excited state behavior of these and closely related compounds [23](b).

Although similar in all these features, Figure 6 illustrates a striking difference between the short (1) and the longer (2 and 3) PSB models. This also appears by comparing the MEP of Figure 3 with the ones shown in Figures 4 and 5: an increasingly pronounced energy plateau emerges along the photoisomerization path as the length of the conjugated chain is extended (see the framed regions in Figures 3, 4, and 5). Thus, while the qualitative features of the $S_{1}$ PES remain the same for all the models, a difference in the dynamics is expected as increasing the length. Due to the reduced steepness (at the SP structure) along the twisting mode, a much longer $S_{1}$ lifetime is expected for model 2 and $\mathbf{3}$ as compared to $\mathbf{1}$. In fact, the system is funneled to the CI much more efficiently in the short PSB, and IVR from totally symmetric (stretching) modes to non totally symmetric (twisting) modes may occur in a subpicosecond timescale [23](d). In fact, the pronounced negative curvature at SP immediately couples the symmetric stretching to the asymmetric twisting. On the other hand, models 2 and $\mathbf{3}$ undergo many oscillations upon the flat $S_{1}$ energy region in order to populate the active (twisting) modes, leading to the funnel (see trajectories in Figure 6). Therefore, retardation in the photoisomerization process is expected [23](b).

Validation of 3 as a realistic model for the 11-cis retinal chromophore (PSB11) of rhodopsin comes from the energy and charge data computed along the photoisomerization path. The computed absorption maximum (482 nm at planar FC) and fluorescence maximum (594$614 \mathrm{~nm}$, depending on the selected point on the MEP along the energy plateau) compare reasonably well with the observed values in hexane solution (458 and $620 \mathrm{~nm}$ respectively) and suggest that the general structure of the energy surface for the isolated cation 3 may be similar to that of PSB11 in solution. Moreover, the computed $\mathrm{S}_{0}-\mathrm{S}_{1}$ dipole moment change $|\Delta \mu|$ (14.0 Debyes) matches very well the observed value (12.0 Debyes), and 

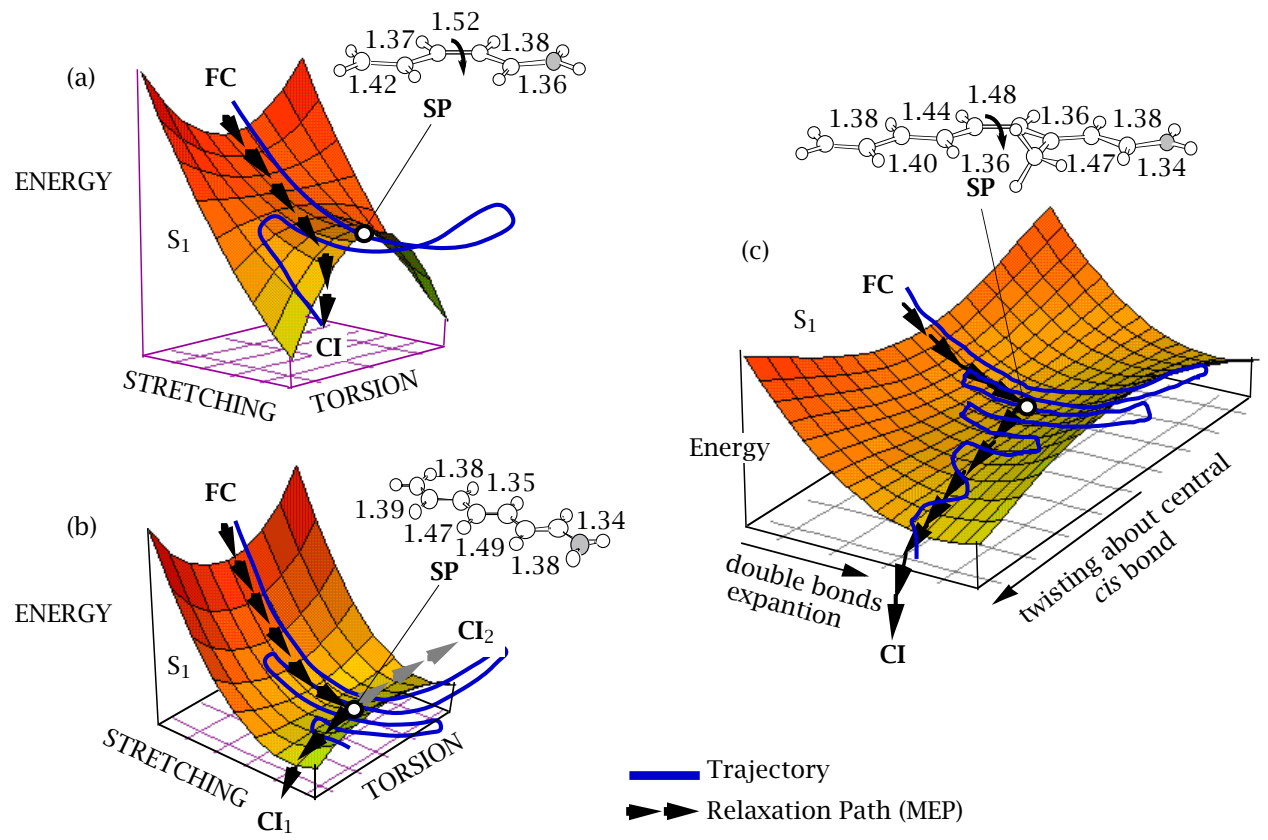

Figure 6. Shape of the $\mathbf{F C} \rightarrow \boldsymbol{S P}$ region of the $\mathrm{S}_{1}$ energy surface for $\mathbf{1}(\mathrm{a}), \mathbf{2}(\mathrm{b})$, and $\mathbf{3}$ (c). The structures of the relaxed planar stationary points (SP) are shown (geometrical parameters in $\AA$ and degrees).

support the strong ionic character of the $S_{1}$ state versus the covalent nature of $S_{0}$ and $S_{2}$. A further evidence for the high quality of model 3 comes from a recent simulation of the resonance Raman (RR) spectra [23](e). As it has been shown, the computed frequencies and RR intensities are in strong agreement with that observed for PSB11 and its isotopomers in solution [23](e), which support the high quality of the simulated $S_{1}$ force field.

Figure 6 provides also a clear example of what has been stated in Section 2.3. The MEP does not travel through the optimized planar transition state SP but, due to the negative curvature at this point, it deviates from the planar-symmetric path and is funneled toward the crossing point along the active twisting mode, before reaching SP. This situation is particularly evident for big negative curvatures such as in 1 (Figure 6(a)). In 2 and (even more) 3 (see Figure 6(b),(c)), the flatness of the energy surface at SP accounts for an initial symmetric MEP that evolves (via a sharp$90^{\circ}$ curve) along the asymmetric twisting mode only in the close vicinity of SP. Thus, while SP allows us to characterize the topology of the $S_{1}$ PES, the system does not travel through this point and the information we get from MEP mapping and geometry optimizations are complementary. The same is for the funnel point: the optimized lower-energy crossing point is different from the conical intersection structure (CI) intercepted by the MEP. While, in general, the difference (both in energy and geometry) may be not too big, the point where decay actually occurs is more correctly the latter.

\section{CONCLUSIONS: THE “TWO-STATE, TWO-MODE” MODEL}

The computational results illustrated above provide a breakthrough into radiative to kinetic energy conversion (i.e. photoisomerization) for RPSBs. These results, in concert with very recent experimental evidences, call for a revision of the models previously proposed for the primary event in rhodopsin proteins, and support a two-state/two-mode model for the photoisomerization of retinal chromophores [23](f).

Model 3 has been proved to give an accurate description of the photochemical behavior of PSB11 in isolated conditions (e.g. in solution), reproducing, with remarkable accuracy, both the force field and the energetic of the excited state in the FC region. The presence of a barrierless path, and a CI structure with the properties of a TICT state is consistent with an ultrafast photoisomerization process with high quantum yields, as indeed observed both in solution and protein. Moreover, the competing channels computed in 2 provide a rational for the observed competitions in the isomerization of internal double bonds as seen in solution for RPSBs. On the other hand, the shape of the $\mathrm{S}_{1} \mathrm{PES}$ and the two-component photoisomerization coordinate suggest the dynamic behavior illustrated in Figure 6(c), where a metastable species performs many skeletal oscillations along an energy plateau before the reactive torsional mode gets fully populated (i.e. IVR has to occur). This metastable species may be assigned to the picosecond "fluorescent-state" observed in 


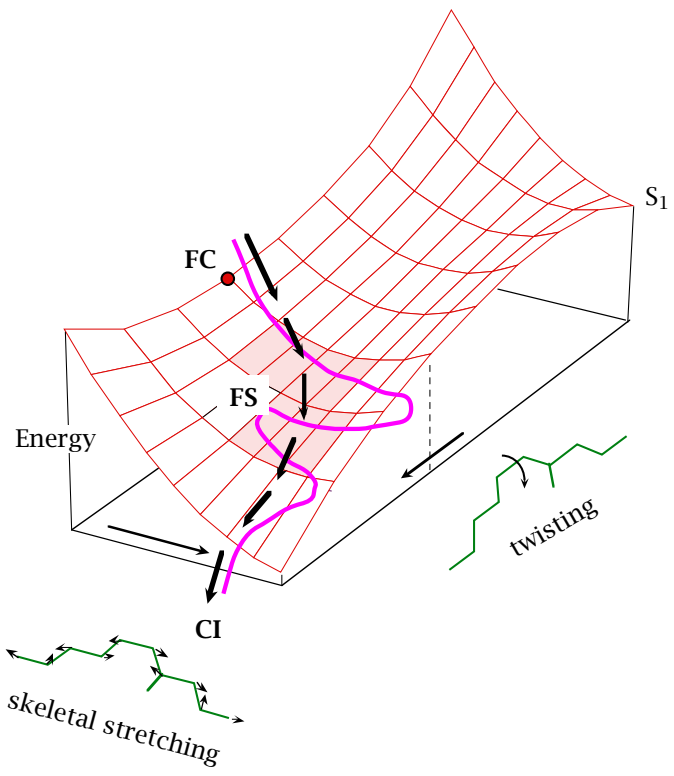

Scheme 4 .

solution. In conclusion, an initial in-plane excited-state relaxation (i.e. symmetric stretching) towards $\mathbf{S P}$ is expected: this has been very recently observed both in solution and protein using modified (i.e. locked) chromophores [24]. Finally, the preserved ionic nature of $\mathrm{S}_{1}$ all along the excited state path calls for a two-state model in the description of the photoisomerization process.

The sub-picosecond (200 fs) timescale observed for the reaction into the protein environment [4](b) is not compatible with the energy plateau found at SP and suggests that the structure of the $S_{1}$ PES given in Figure 6(c) may be modified by the protein cavity to yield a less stable SP region. Such a situation may arise if the energy plateau is reduced or suppressed as shown in Scheme 4 . Here, the surface is made asymmetric with respect to the twisting mode by, for example, the chiral environment, and/or charged and dipolar groups of the protein cavity. Thus, coupling between stretching and twisting modes may already be active during initial motion out of the FC region (as in 1) making the fluorescent state (FS) very short-living [23](e).

We hope we have given a convincing demonstration that it is technically feasible to describe a photochemical reaction co-ordinate, from energy absorption to photoproduct formation. The conceptual problems that need to be understood in order to apply quantum chemistry to photochemical problems relate mainly to the characterization of the conical intersection funnel and the computation of MEPs. Obviously, any mechanistic study undertaken using quantum chemistry methods requires considerable physical and chemical insight. As for a thermal reaction there is no method that will generate automatically all the possible mech- anistic pathways that might be relevant, thus in excited state chemistry one still needs to apply skills of chemical intuition and make sensible hypotheses that can then be explored computationally. This can be even more difficult, since more electronic states are involved. Anyway, as illustrated by the examples above, new interesting information may be collected leading to a novel reactivity view for photochemical processes.

\section{References}

[1] (a) H. J. C. Jacobs and E. Havinga, Advances in Photochemistry, J. N. Pitts Jr., G. S. Hammond, and K. Gollnick (eds.), Vol. 11, John Wiley \& Sons, New York, 1979, pp. 305-373.

(b) W. G. Dauben, E. L. McInnis, and D. M. Mincho, Rearrangements in ground and excited states, P. De Mayo (ed.), Vol. 3, Academic Press, London, 1980, pp. 91-129.

[2] H. G. Heller, C. C. Elliot, K. Koh, S. Al-Shihry, and J. Whittall, Photochemistry and Polymeric Systems, J. M. Kelly, C. B. Mc Ardle, and M. J. de F. Maunder (eds.), The Royal Society of Chemistry, London, 1993, pp. 156-168.

[3] T. Yoshizawa and O. Kuwata, CRC Handbook of Organic Photochemistry and Photobiology, W. M. Horspool and P.-S. Song (eds.), CRC Press, Boca Raton, FL, 1995, pp. 1493-1499.

[4] (a) H. Kandori, Y. Katsuta, M. Ito, and H. Sasabe, J. Am. Chem. Soc. 115 (1995), 2669.

(b) Q. Wang, R. W. Schoenlein, L. A. Peteanu, R. A. Mathies, and C. V. Shank, Science 266 (1994), 422.

[5] A. H. Zewail, J. Phys. Chem. 100 (1996), 12701.

[6] M. O. Trulson and R. A. Mathies, J. Phys. Chem. 94 (1990), 5741.

[7] P. J. Reid, S. J. Doig, S. D. Wickham, and R. A. Mathies, J. Am. Chem. Soc. 115 (1993), 4754, and references cited therein.

[8] S. Pullen, L. A. Walker II, B. Donovan, and R. J. Sension, Chem. Phys. Lett. 242 (1995), 415.

[9] D. R. Cyr and C. C. Hayden, J. Chem. Phys. 104 (1996), 771.

[10] D. G. Truhlar and M. S. Gordon, Science 249 (1990), 491.

[11] (a) A. Gilbert and J. Baggott, Essentials of Molecular Photochemistry, Blackwell Scientific Publications, Oxford, 1991.

(b) J. Michl and V. Bonacic-Koutecky, Electronic Aspects of Organic Photochemistry, Wiley, New York, 1990.

(c) M. Klessinger and J. Michl, Excited States and Photochemistry of Organic Molecules, VCH Publishers, New York, 1994.

(d) B. O. Roos, Adv. Chem. Phys. (Ab Initio Methods in Quantum Chemistry-II), K. P. Lawley (ed.), Vol. 69, Wiley, New York, 1987, pp. 399-446. 
(e) M. Desouter-Lecomte and J. C. Lorquet, J. Chem. Phys. 71 (1979), 4391.

(f) F. Bernardi, M. Olivucci, and M. A. Robb, Chem. Soc. Rev. 25 (1996), 321.

(g) F. Bernardi, M. Olivucci, J. Michl, and M. A. Robb, The Spectrum 9 (1997), 1.

(h) D. R. Yarkony, Acc. Chem. Res. 31 (1998), 511.

(i) D. R. Yarkony, J. Phys. Chem. 100 (1996), 18612.

[12] W. T. A. M. Van der Lugt and L. J. Oosteroff, J. Am. Chem. Soc. 91 (1969), 6042.

[13] E. Teller, Isr. J. Chem. 7 (1969), 227.

[14] H. E. Zimmerman, J. Am. Chem. Soc. 88 (1966), 1566.

[15] J. Michl, Mol. Photochem. 4 (1972), 243.

[16] W. Fuss, K. L. Kompa, S. Lochbrunner, and A. M. Muller, Chem. Phys. 232 (1998), 174.

[17] Gaussian 98, Revision A.6, M. J. Frisch, G. W. Trucks, H. B. Schlegel, G. E. Scuseria, M. A. Robb, J. R. Cheeseman, V. G. Zakrzewski, J. A. Montgomery Jr., R. E. Stratman, J. C. Burant, S. Dapprich, J. M. Millam, A. D. Daniels, K. N. Kudin, M. C. Strain, O. Farkas, J. Tomasi, V. Barone, M. Cossi, R. Cammi, B. Menucci, C. Pomelli, C. Adamo, S. Clifford, J. Ochterski, G. A. Petersson, P. Y. Ayala, Q. Cui, K. Morokuma, D. K Malick, A. D. Rabuck, K. Raghavachari, J. B. Foresman, J. Cioslowski, J. V. Ortiz, B. B. Stefanov, G. Liu, A. Liashenko, P. Piskorz, I. Komaromi, R. Gomperts, R. L. Martin, D. J. Fox, T. Keith, M. A. Al-Laham, C. Y. Peng, A. Nanayakkara, C. Gonzalez, M. Challacombe, P. M. W. Gill, B. G. Johnson, W. Chen, M. W. Wong, J. L. Andres, C. Gonzalez, M. Head-Gordon, E. S. Replogle, and J. A. Pople, Gaussian, Inc., Pittsburgh PA, 1998.

[18] MOLCAS, Version 4. K. Andersson, M. R. A. Blomberg, M. P. Fülscher, G. Karlström, R. Lindh, P.-Å. Malmqvist, P. Neogrády, J. Olsen, B. O. Roos, A. J. Sadlej, M. Schütz, L. Seijo, L. Serrano-Andrés, P. E. M. Siegbahn, and P.-O. Widmark, Lund University, Sweden, 1997.

[19] (a) J. J. McDouall, K. Peasley, and M. A. Robb, Chem. Phys. Lett. 148 (1988), 183. (b) K. Andersson, P. A. Malmqvist, and B. O. Roos, J. Chem. Phys. 96 (1992), 1218.

[20] C. Gonzalez and H. B. Schlegel, J. Phys. Chem. 94 (1990), 5523.

[21] (a) H. B. Schlegel, Ab Initio Methods in Quantum Chemistry, K. P. Lawley (ed.), Vol. 1, John Wiley \& Sons Ltd., New York, 1987, pp. 249-286, and references cited therein.

(b) J. B. Foresman and F. Aeleen, Exploring Chemistry with Electronic Structure Methods, Gaussian, Inc., Pittsburgh, 1996, Chapt. 3, and references cited therein.

[22] (a) P. Celani, M. A. Robb, M. Garavelli, F. Bernardi, and M. Olivucci, Chem. Phys. Lett. 243 (1995), 1.

(b) M. Garavelli, P. Celani, M. Fato, M. Olivucci, and M. A. Robb, J. Phys. Chem. A 101 (1997), 2023.

[23] (a) M. Garavelli, P. Celani, F. Bernardi, M. A. Robb, and M. Olivucci, J. Am. Chem. Soc. 119 (1997), 6891.

(b) M. Garavelli, F. Bernardi, M. Olivucci, T. Vreven, S. Klein, P. Celani, and M. A. Robb, Faraday Discuss. 110 (1998), 51.

(c) M. Garavelli, T. Vreven, P. Celani, F. Bernardi, M. A. Robb, and M. Olivucci, J. Am. Chem. Soc. 120 (1998), 1285.

(d) T. Vreven, F. Bernardi, M. Garavelli, M. Olivucci, M. A. Robb, and H. B. Schlegel, J. Am. Chem. Soc. 119 (1997), 12687.

(e) M. Garavelli, F. Negri, and M. Olivucci, J. Am. Chem. Soc. 121 (1999), 1023.

(f) R. Gonzalez-Luque, M. Garavelli, F. Bernardi, M. Merchan, M. A. Robb, and M. Olivucci, Proc. Nat. Acad. Sci. USA 97 (2000), 9379.

[24] (a) T. Ye, N. Friedman, Y. Gat, G. H. Atkinson, M. Sheves, M. Ottolenghi, and S. Ruhman, J. Phys. Chem. B 103 (1999), 5122.

(b) T. Ye, E. Gershgoren, N. Friedman, M. Ottolenghi, M. Sheves, and S. Ruhman, Chem. Phys. Lett. 314 (1999), 429. 


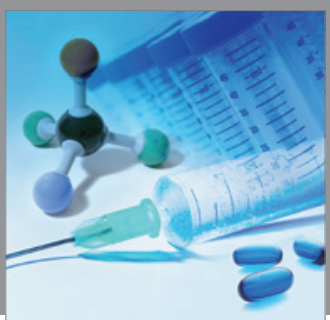

International Journal of

Medicinal Chemistry

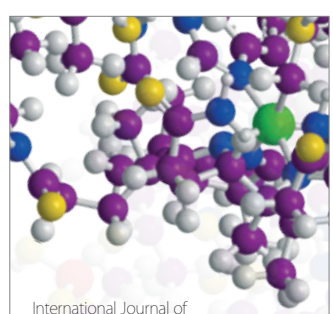

Carbohydrate Chemistry

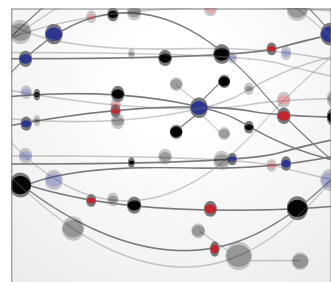

The Scientific World Journal
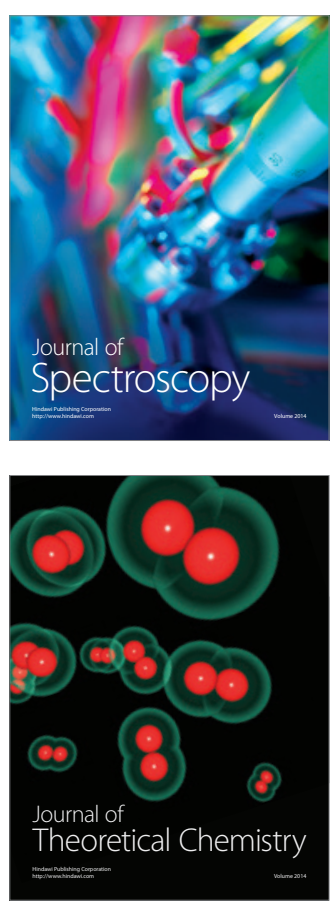
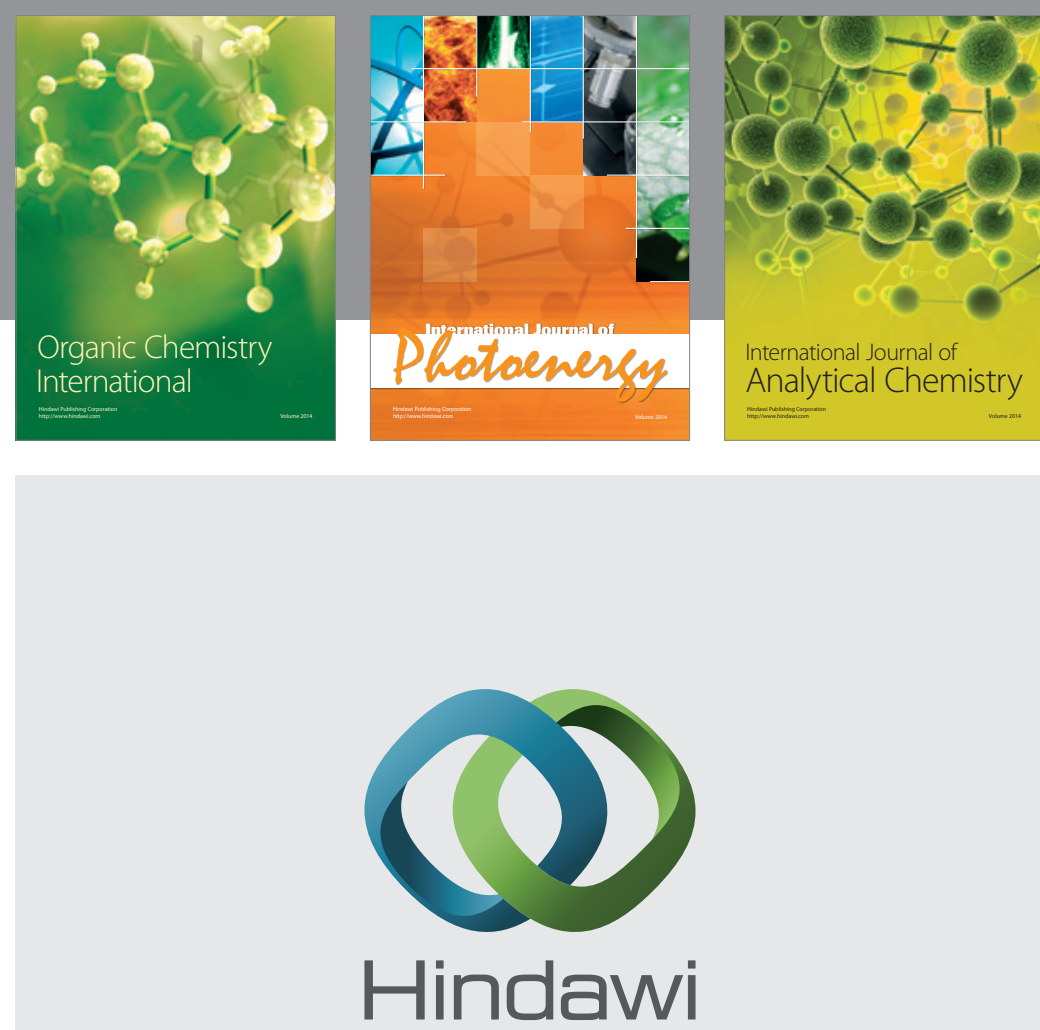

Submit your manuscripts at

http://www.hindawi.com
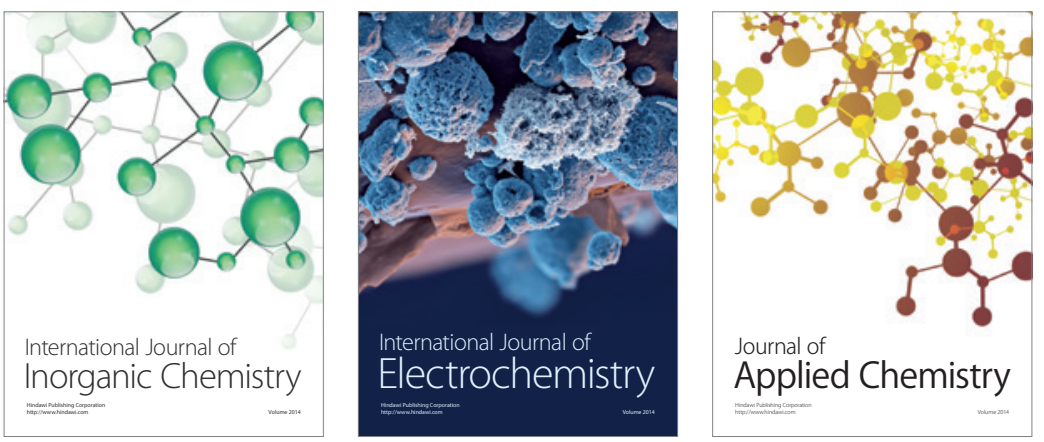

Journal of

Applied Chemistry
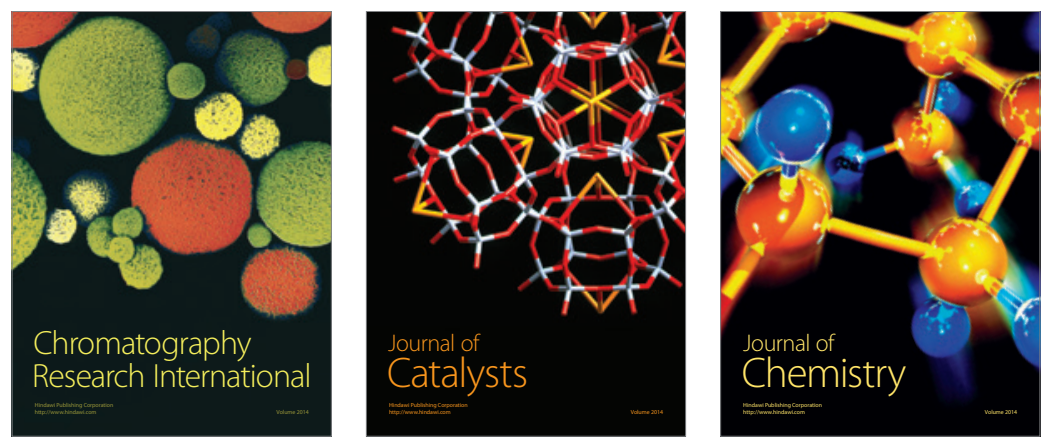
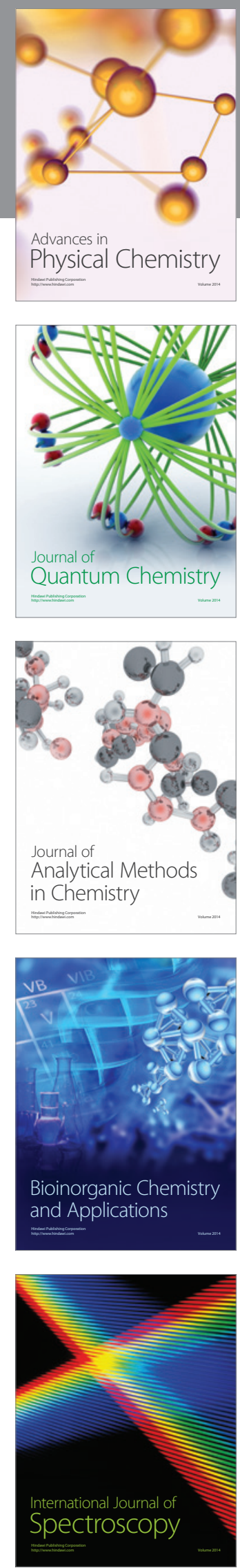